\title{
Evaluation and orientations to Grade 10 mathematics in schools differentiated by social class
}

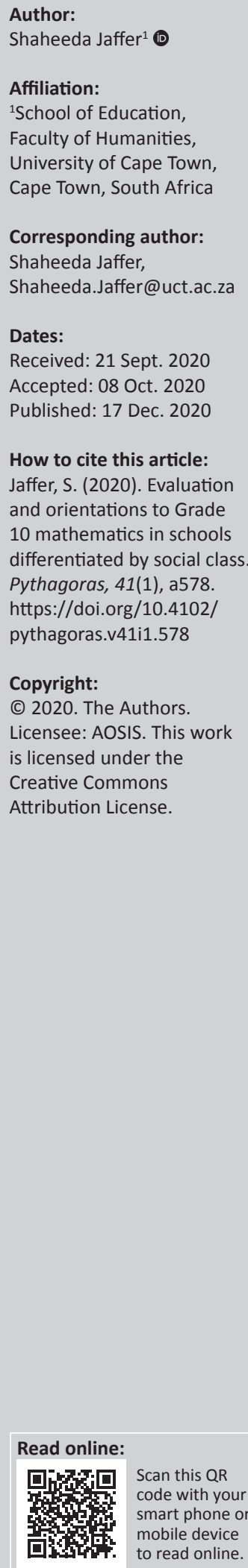

In South Africa, differential performance in school mathematics with respect to social class remains an enduring concern as reflected in national and international large-scale assessments. The article examines the implications of evaluation for orientations to mathematics in a school populated by learners from upper-middle-class or elite backgrounds and a school populated by learners from working-class backgrounds. The particular focus is on mathematics problems featured in tests used by two Grade 10 teachers in each school and teachers' marking of learners' test scripts. A distinction between single-topic and multi-topic mathematics problem types is refracted through an analysis that draws on the adaptation by Davis of Lotman's distinction between content orientation and expression orientation with respect to the reproduction of texts.

The analysis reveals a preponderance of single-topic mathematics problems and the absence of multi-topic mathematics problems employed in the school populated by learners from working-class backgrounds and the presence of both single-topic and multi-topic problem types in the school populated by learners from middle-class or elite backgrounds. Differences in the types of mathematical problems suggest differences in mathematical demand expected of learners and differences in their preparation for examinations in the two social class contexts.

The selection of test problems and the marking of test scripts as instances of evaluation construct an orientation to mathematics that is expression oriented in the working-class context whereas both expression and content orientations are evident in the middle-class or elite context. The analysis provides a potential explanation for the persistent disparity in mathematics performance along social class lines in South African secondary schools.

Keywords: evaluation, marking, orientation to mathematics, content orientation, expression orientation, problem types, social class; computational resources.

\section{Introduction}

Narrowing the achievement gap in school mathematics in South Africa with respect to social class remains a persistent social justice issue in spite of extensive curriculum reforms. Difference in school mathematics performance between learners from middle-class families and learners from working-class families in South Africa has to a great extent been documented in large-scale quantitative studies (e.g., Reddy, Van der Berg, Lebani, \& Berkowitz, 2006; Spaull \& Kotze, 2015).

We have to bear in mind though that social class and 'race' remain intertwined in South Africa. Despite 'racial' desegregation of more affluent schools, poorer schools remain populated largely by 'Black' learners (Spaull, 2019). Thus, national assessments (e.g. matric examinations) and international assessments (e.g. Third International Mathematics and Science Study, TIMSS) continue to reflect both a social class and 'racial' achievement gap in school mathematics (Spaull, 2019).

Some smaller qualitative studies are concerned with the nature of the relation between mathematics performance and social class in an attempt to understand the underlying factors impacting on learner performance in school mathematics. Most research in mathematics education in South Africa (e.g. Carnoy et al., 2011; eds. Graven \& Venkat, 2017; Schollar, 2008) tends to focus on learners from working-class families as part of efforts aimed at closing the social class performance gap (also see Graven, 2014) since those learners constitute the majority under-performing in mathematics. There are a few exceptions, studies focusing on learners from working-class families, particularly 'Black' learners in schools previously 
intended for 'White' learners only (e.g. Feza, 2012; Swanson, 2002, 2006). Furthermore, there are a limited number of South African comparative studies of school mathematics in pedagogic contexts differentiated with respect to learners' social class membership (Hoadley, 2007; Jaffer, 2018; Jaffer \& Davis, 2012). Thus, little is known about the pedagogic practices with regard to school mathematics in upper-middle-class or elite schools, particularly secondary schools. This article aims to contribute to our knowledge of the pedagogic practices in mathematics classrooms in a school populated by learners from uppermiddle-class or elite families which is compared to a school populated by learners from working-class families.

Despite diversity with respect to methodology, there is convergence in the findings of local and international comparative studies concerned with school mathematics in relation to the differential distribution of knowledge across different social class settings (Anyon, 1980, 1981; Atweh, Bleicher, \& Cooper, 1998; Atweh \& Cooper, 1995; Cooper \& Dunne, 2000; Hoadley, 2005, 2007; O’Halloran, 2004).

The orientation to mathematics in schools populated by learners from working-class families is often described in this literature as procedural or weakly bounded from the 'everyday'. In contrast, orientation to mathematics in schools populated by learners from middle-class families is commonly described in this literature as conceptual as opposed to procedural or as strongly bounded from the 'everyday'. The literature thus partitions descriptions of mathematics realised in pedagogic contexts differentiated with respect to social class in terms of the academic-everyday distinction and procedural-conceptual opposition and remains relatively silent on similarities in the mathematics realised in those pedagogic contexts.

This article examines the orientations to mathematics in two schools differentiated with respect to learners' social class membership by examining how evaluation structures orientation to mathematics. In particular, the research question pursued in this article is: How does evaluation function in relation to Grade 10 mathematics in two schools that differ with respect to the social class membership of their learner populations and what are the implications for learners' orientations to mathematics?

\section{Theoretical framework}

The general methodology underpinning the analysis of data in this article is informed by Bernstein's (2000) notion of the pedagogic device, specifically in relation to evaluation. The functioning of evaluation in the two social class pedagogic contexts is examined through the selection of test problems and the marking of learners' test scripts. The orientations to mathematics constructed by the way in which evaluation functions in a pedagogic context is explored further through Davis's (2011) appropriation of Lotman's (1990) notions of content orientation and expression orientation.

\section{Pedagogic evaluation}

Bernstein (2000) argues that evaluation is central to pedagogy. He uses the term evaluation as opposed to assessment to signal that evaluation is a broader notion that encompasses anything that marks out criteria for the recognition and realisation of legitimate knowledge statements in a particular pedagogic situation. Evaluation can be instantiated, for example, in a range of activities such as the marking of a learner's work, teacher's responses to learners' responses to teacher questions, teachers' explanations or learners' solutions to mathematics problems (Davis, 2005).

Pedagogy entails a relationship between two or more notional pedagogic subjects, the teacher and the learner, with the reproduction of knowledge being the knot that ties the two together, referred to as a didactic relation by Chevallard (1989, p. 4). Teachers and learners relate to knowledge in different ways. The teacher reproduces knowledge in order to communicate what they want learners to produce and how they should produce what counts as legitimate knowledge in a pedagogic situation. The learner always asks themselves what the teacher expects of them and how should they achieve what the teacher wants from them. The relationship between the teacher and learner is therefore essentially evaluative in that what they produce is structured by evaluation. As such, evaluation is essential for the pedagogic reproduction of knowledge.

For Bernstein, the centrality of evaluation is emphasised in his discussion of the pedagogic device, which serves as an analytic and descriptive resource for describing the transformation of knowledge into pedagogic communication:

We can see that key to pedagogic practice is continuous evaluation. [...] This is what the device is about. Evaluation condenses the meaning of the whole device. We are now in a position where we can derive the whole purpose of the device. The purpose of the device is to provide a symbolic ruler of consciousness. (Bernstein, 2000, p. 50)

The pedagogic device, which is Bernstein's attempt to relate social structure to individual consciousness, entails three hierarchically related 'rules' - the distributive, recontextualising and evaluative rules (Bernstein, 2000) which together structure the production and reproduction of knowledge. The evaluative rule is derived from the recontextualising rule, which is in turn derived from the distributive rule. The distributive rule 'mark $[s]$ and distribute[s] who may transmit what to whom' (Bernstein, 1990, p. 158). The distributive rule, which regulates the distribution of 'forms of knowledge, forms of consciousness and forms of practice to social groups' (Bernstein, 1990, p. 42), plays a key role in the reproduction of the social division of labour by distributing access to social goods, contributing to the reproduction of patterns of social relations. The recontextualising rule, which governs the selection of 
knowledge from the field of production and other discourses such as theories of learning and teaching, for the formation of pedagogic discourse (e.g. school mathematics), creates specialised pedagogic subjects (Bernstein, 1996, p. 46).

The evaluative rule is key in the pedagogic reproduction of knowledge. Bernstein argues that pedagogic practice is characterised by the ever-present evaluative activity where evaluation distinguishes legitimate from non-legitimate knowledge statements for learners (Bernstein, 1996, p. 50). Note that legitimate knowledge is not necessarily knowledge that is accepted as correct in general. Legitimate knowledge is that sanctioned in a specific pedagogic situation. For example, when a teacher, accepts 2 as the only solution to the equation $x^{2}=4$ where $x \in \mathbb{R}$, then incorrect knowledge is accepted as legitimate. It is also possible that correct mathematical knowledge could be considered non-legitimate in a pedagogic situation.

Texts produced by teachers and learners make explicit the knowledge accepted as legitimate in a pedagogic situation and provide criteria that mark out legitimate knowledge statements from non-legitimate statements. The centrality of evaluation is underscored by Bernstein (1996) when he declares that 'evaluation condenses the meaning of the device' (p. 50). Bernstein's claim implies that both the recontextualising rule as well as the distributive rule are entailed in the evaluative rule. Researchers, however, sometimes construct their accounts of pedagogic situations in the reverse order by examining how evaluation functions in pedagogic situations to reveal the content of the recontextualising rules from which the distributive rules are derived. This article is concerned with the functioning of evaluation and its structuring effect on orientations to mathematics in pedagogic contexts.

\section{Orientation to mathematics}

Lotman's distinction between text-oriented cultures and grammaroriented cultures has been usefully appropriated for describing orientations to mathematics (see Davis, 2011; Davis \& Ensor, 2018; Dowling, 1998; Jaffer, 2010, 2018). Lotman describes grammar-oriented and text-oriented societies as follows:

Cultures can be governed by a system of rules or by a repertoire of texts imposing models of behaviour. In the former category, texts are generated by combinations of discrete units and are judged correct or incorrect according to their conformity to the combinational rules. In the latter category, society directly generates texts, which constitute macro units from which rules can eventually be inferred, but which initially and most importantly propose models to be followed and imitated.

A grammar-oriented culture [i.e. governed by a system of rules] depends on 'Handbooks', while a text-oriented culture [i.e. governed by a repetition of model texts] depends on 'The Book'. A handbook is a code which permits further messages and texts, whereas a book is a text, generated by an as-yet-unknown rule which, once analyzed and reduced to a handbook-like form, can suggest new ways of producing further texts (Eco in Lotman, 1990, p. xi, italics in original).
A grammar-oriented culture which is governed by a system of rules is juxtaposed with a text-oriented culture which is governed by a repetition of model texts. Lotman's categories, while useful as a heuristic for thinking about pedagogy, require adaptation for analysis of pedagogic situations. Pedagogic modalities in which learners are encouraged to reproduce texts, through repetition and rehearsal, that precisely conform with texts considered as legitimate in the pedagogic context, are suggestive of Lotman's text-oriented cultures. The use of 'model answers' for classes of mathematics problems often used in pedagogic contexts resonates with Lotman's text orientation.

His concept of grammar-oriented cultures, on the other hand, is comparable with pedagogic modalities that encourage 'syntactical symbol manipulation and propositional descriptions of relations between mathematical objects' (Davis, 2011, p. 315). For example, a grammar orientation is in play with the recognition that the vertical column method of two-digit addition and horizontal method of two-digit addition which explicitly involves partitioning two-digit numbers into tens and units are governed by the same mathematical structure (addition over natural numbers) despite differences at the level of expression.

Although combinatorial rules are embodied in model texts in text-oriented cultures, the repetition and rehearsal of texts seems to suggest that individuals can produce texts without the use of combinatorial rules (see Davis, 2011). Davis (2011) argues that this opposition cannot apply in pedagogic situations because, following Chomsky (2009), combinatorial rules are essential components of thought and language and so of mathematics, and so ought to be present in textoriented pedagogic situations. Davis (2011, pp. 316-317) adopts Lotman's distinction between expression orientation and content orientation as a more appropriate distinction for describing aspects of the realisation of mathematics in pedagogic situations, given that Lotman aligns text orientation with expression orientation and grammar orientation with content orientation:

Lotman suggests that text-oriented societies are at the same time expression-oriented ones, while grammar-oriented societies are content-oriented. The reason for such a definition becomes clear when one considers the fact that a culture which has evolved a highly differentiated content-system has also provided expression-units corresponding to its content-units, and may therefore establish a so-called 'grammatical' system - this simply being a highly articulated code. On the contrary a culture which has not yet differentiated its content-units expresses (through macroscopic expressive grouping: the texts) a sort of content-nebula (Eco, 1976, p. 138, italics in original).

So, for Davis (2011, pp. 316-317), both expression orientation and content orientation are combinatorial. An expression orientation refers to an orientation to mathematics that focuses primarily on the expressive elements required and entails a system of combinatorial rules that operates directly on the expressive elements to generate texts (Davis, 2011). The 'change sides, change signs' rule used to solve equations 
is an example of combinatorial rules focusing on the expressive elements of an equation, allowing an individual to solve an equation without the notion of an equation being present. For example, the equation $2 x+1=5$ can be solved by moving the symbols ' +1 ' from the left-hand side to the right-hand side, and in doing so changing to the symbols ' -1 '. Thus, combinatorial rules operate directly on the symbols. Furthermore, repetition and rehearsal of texts that precisely imitate texts considered as legitimate in a pedagogic situation is suggestive of an expression orientation. For example, a teacher's model solution to the equation $(x+1)^{2}=9$ which insists that the equation must be transformed into standard form $\left(a x^{2}+b x+c=0\right)$ before it can be solved is promoting an expression orientation because the teacher's solution focuses on the form of expression. The equation $(x+1)^{2}=9$ can be solved by using square roots, thus bypassing the need to transform $(x+1)^{2}=9$ into standard form

With a content orientation, the expressive elements are secondary, functioning merely as resources for communicating mathematics (Davis, 2011). Operating with a content orientation implies using combinatorial rules that abide by mathematical structures. For example, an individual who produces 4 as the solution to the equation $2 x+1=9$ as follows: $2 x+1=9 \Leftrightarrow 2 x+1=8+1 \Leftrightarrow 2(4)+1$ is using the notion of equality and the right cancellation theorem as computational resources situated in the field of the reals as opposed to the 'change signs, change sides' rule which is located outside of the field of the reals.

\section{Mathematics problems}

Previous studies show that a large proportion of mathematics lessons is spent on solving mathematics problems (see, for example, US Department of Education, 2003). Mathematics problems are commonly used as the main vehicles for the elaboration of mathematics topics in schooling. Typically, teachers use mathematics problems as worked examples to illustrate particular solution procedures, as practice exercises to provide opportunities for learners to become proficient at executing those procedures and in assessment tasks such as tests and examinations to ascertain learners' knowledge of mathematics. Mathematics problems, sometimes referred to as mathematics tasks in the literature (see eds. Shimizu, Kaur, Huang, \& Clarke, 2010), entail the use of computations to solve problems. Arithmetic problems, solving equations, sketching graphs or solving geometric riders are examples of mathematics problems.

Mathematics tasks or problems are described in different ways in the literature. Boaler's (1998) distinction between 'open-ended' and 'closed' mathematics tasks is essentially a distinction between conceptual and procedural tasks (see also Kaur, 2010). The distinction between 'realistic' and 'esoteric' problems (e.g. Cooper \& Dunne, 2000) or categorisation of mathematics tasks as 'authentic' or 'realworld' (e.g. Kaiser \& Schwarz, 2010) can be aligned with the academic-everyday distinction. The Curriculum Assessment Policy Statement (CAPS) Further Education and Training assessment guidelines stipulate the following distribution of problem types: knowledge $(20 \%)$, routine procedures $(35 \%)$, complex procedures $(30 \%)$ and problem solving (15\%) (Department of Basic Education, 2011, p. 53).

The categorisation of problem types used in this article references the topics indexed by the mathematics problems because of the larger project's interest in the announced topic and the content associated with the topic. The announced topic refers to a name used by teachers, learners and textbooks to indicate a particular selection of mathematics contents. It should be noted that the content associated with announced topics can only be revealed when teachers and learners solve mathematics problems. Chitsike (2011), Davis (2013) and Jaffer $(2012,2018)$ provide examples of studies that examine the computations employed by teachers and learners when solving mathematics problems to ascertain the content associated with topics. They reveal that the realised content in the name of a topic is not stable across pedagogic contexts and that the content realised in relation to a topic could be aligned with or diverge from the content associated with the topic from the point of view of the field of mathematics. The example related to the solving of linear equations discussed above illustrates this point. The use of the 'change sides, change signs' rule to solve linear equations is an example where content associated with the topic diverges from the content associated with the topic from the point of view of the field of mathematics whereas the use of the right cancellation theorem to solve a linear equation is illustrative of content that aligns with the content associated with the topic from the point of view of the field of mathematics.

I distinguish between single-topic mathematics problems and multi-topic mathematics problems. A mathematics problem that indexes one announced topic is referred to as a single-topic problem, typically accompanied by problem statements such as 'sketch the parabola' or 'calculate the equation of the function'. Multi-topic mathematics problems involve more than one announced topic. Figure 1 references five announced topics: (1) graphing parabola (Problems 1.1, 1.2 and 1.3), (2) graphing a linear function (Problem 1.3),

\begin{tabular}{|c|c|c|}
\hline Grade 10 & \multirow{2}{*}{$\begin{array}{l}\text { Common test functions } 2012 \\
f(x)=-2 x^{2}+2 x+4 \text { and } g(x)=-8 x+4\end{array}$} & Total 45 marks \\
\hline 1. Given & & \\
\hline 1.1 & Determine the co-ordinates of the $x$-intercepts of graph $f$. & (3) \\
\hline 1.2 & Determine the co-ordinates of the turning point of $f$. & (3) \\
\hline 1.3 & $\begin{array}{l}\text { Draw the graphs of } f(x)=-2 x^{2}+2 x+4 \text { and } g(x)=-8 x+4 \text { on } \\
\text { the same set of axes on your answer sheet. Label the } \\
\text { graphs clearly, including all the intercepts with the axes } \\
\text { and the turning point. }\end{array}$ & (5) \\
\hline 1.4 & Write down the range of $f$. & (1) \\
\hline 1.5 & $\begin{array}{l}\text { Determine algebraically the coordinates of the points of } \\
\text { intersection of } f(x) \text { and } g(x)\end{array}$ & (5) \\
\hline 1.6 & Use your graphs to determine for which values of $x, f(x)<0$. & $(2)$ \\
\hline
\end{tabular}

FIGURE 1: Problem 1 of test administered by Ivory College teachers. 
(3) range of functions (Problem 1.4), (4) points of intersection (Problem 1.5) and (5) graphic inequalities (Problem 1.6). Each of the problems 1.1 to 1.6 is classified as a singletopic mathematics problem but together they constitute a multi-topic problem because Problem 1 deals with more than one topic.

Multi-topic problems encourage inter-topic connectivity because they require learners to use previously encountered computational resources such as definitions, propositions and procedures together with the newly introduced knowledge to solve mathematics problems and so connect new topics to topics dealt with previously.

However, the content realised through solving mathematics problems is not the issue under discussion in this article. Instead, mathematics problems serve as instances of pedagogic evaluation in that they are selected by teachers for the elaboration of mathematics topics and are used to assess learners' acquisition of mathematics topics.

\section{Social class and 'race'}

Social class serves as a background contextual variable in this study and not as an explanatory category. The intention here is not to set up causal relations between social class on the one hand and orientations to mathematics on the other hand. The selection of empirical sites on the basis of the social class membership of a school's learner population was guided by the assumption that because social class continues to be aligned with differential mathematics achievement, such differences potentially point to contrasts in the way evaluation functions.

As indicated earlier 'race' and social class remain inextricably linked in South Africa. I use the term 'race' in quotation marks because 'race' has little biological validity (Yudell, Roberts, DeSalle, \& Tishkoff, 2016). 'Racial' categories were imposed on South African citizens during apartheid and are not necessarily accepted by individuals categorised as such. I use the apartheid categories because this historical legacy has shaped and continues to shape schooling in South Africa. Since 1994, census classifications have distinguished between 'Black African', 'Coloured', 'Indian or Asian' and 'White'. I use the terms 'African', 'Coloured', 'Indian' and 'White' all with the first letter capitalised. The term 'Black' is used as an encompassing term to refer to 'Africans', 'Coloureds', and 'Indians'.

Deregulation of 'race' as an admission requirement in all schools followed shortly after the demise of apartheid. Subsequently, post-apartheid South Africa has witnessed substantial transformation in the 'racial' demographics of school populations. 'White', 'Coloured' and 'Indian' schools have changed with respect to their 'racial' composition, but the learner populations of ex-Department of Education and Training schools have to a large extent remained exclusively 'African' (Sujee, 2004, as cited in Chisholm \& Sujee, 2006).
Enrolment patterns across the South African schooling system are now determined largely by school fees. No-fee and low-fee-paying public and independent schools serve the majority of South African children - working-class and lower-working-class children who are predominantly 'African' and 'Coloured' (Franklin, 2017). Schooling has thus become stratified with respect to social class and remains to a large extent stratified in terms of 'race'.

\section{Research design}

This article reports on a research study (Jaffer, 2018) that is concerned with the functioning of evaluation in Grade 10 mathematics classrooms differentiated with respect to social class. The study was designed as a case study (Yin, 2009). Although Yin (2009) refers to case study as a method of research that allows for in-depth study of a small number of cases where the focus is on understanding a complex phenomenon, I do not treat case study as a specific research method. Instead a 'case' is simply viewed as a means of describing the selection procedure of a study (Brown \& Dowling, 1998, p. 151). The theoretical resources recruited for this article were described above.

The study was designed as a comparative study of two schools that differ with respect to the social class membership of their student population. The selection of schools was based on learners' social class membership. School fees were used as a proxy for learners' social class membership which was confirmed through biographical information obtained from a learner questionnaire and school questionnaire completed by the principal. Arbor High is a no-fee school and Ivory College is a private school with school fees set at R85 000 per annum in 2012.

Ivory College is 'racially' mixed and serves learners from upper-middle-class or elite families. Arbor High is populated by 'African' learners from working-class families. At each school, two teachers and learners in one of their Grade 10 mathematics classes comprise the research participants of the study. Sara and Jada taught at Ivory College, Maya and Jono at Arbor High.

The data collected for the research study included videorecorded mathematics lessons, curriculum and teaching resources such as textbooks, worksheets, tests designed and administered by the teachers, test scripts from the four Grade 10 mathematics classes and interviews with selected learners in each class. This article focuses specifically on the mathematics problems used in a test administered by the teachers and the marking of test scripts as instances of pedagogic evaluation.

The announced topics in the observed lessons of the four teachers are all related to the CAPS curriculum topic Functions, which according to the CAPS pace setter is scheduled for teaching in the second term of the school year (Department of Basic Education, 2011, p. 17). All the lessons were observed in the third term. The tests, based 
on the topics dealt with in observed lessons, were set and conducted by teachers at the schools. At both schools, the tests took place after the observed lessons at a time decided by each school. The tests across the two schools and within Arbor High differed with respect to the announced topics. The analytic categories in relation to problem types and orientations to mathematics are independent of the announced topics.

\section{Ivory College test (Sara and Jada)}

Ivory College wrote a 'common' test, which was a test set by one of the Grade 10 teachers and written by all Grade 10 learners at the school. The test, referred to as Common Test Functions 2012 (see Figure 1), covered linear functions, parabolas, exponential functions and hyperbolas. The test included topics dealt with during the observed lessons as well as topics covered after the observed lessons. The test comprised four problems which are classified as multi-topic mathematics problems because they each involve more than one announced topic.

Ivory College learners were given considerable practice opportunities by their teachers, mostly independently of the teacher, since the tasks were either given as homework exercises or tutorials that were required to be submitted for marks. The test problems in the Common Test Functions 2012 are very similar to the problems contained in the parabola worksheet used during the observed lessons. In addition, learners were given a tutorial on graphs which they were required to submit for marks. The tutorial covered the same topics as the Common Test Functions 2012 and consisted of single-topic problems such as 'sketch the graph on separate axes. Show all intercepts with the coordinate axes, turning points, axes of symmetry and asymptotes' as well as multi-topic problems such as those shown in Figure 1. The graph tutorial and the parabola worksheet therefore served as preparation for the test, which in turn appeared to be intended as preparation for the examination.

In addition, Ivory College learners were exposed to variations in phrasing of mathematics problems. For example, problems on calculating the points of intersection of two functions were posed in different ways in the worksheet and the tutorial: (1) calculate the points of intersection of the functions $f$ and $g$, (2) calculate the values for $x$ for which $f(x)=g(x)$, (3) solve the equation $-x^{2}+9=2 x+6$ and explain how this helps find two of the points labelled on the graph or (4) calculate the coordinates of the point $T$. In the last problem, learners were first expected to work out that point $T$ represents a point of intersection of two functions. Mathematics problems that did not directly name the procedure required to solve the problem was another feature of the worksheet, tutorial and the common test used by Ivory College teachers. Learners were, therefore, expected to analyse the problem statement in order to decipher the appropriate procedure required.
The selection of mathematics problems for the test, worksheet and tutorial suggests that evaluation functions in a way that attempts to move beyond recall and rehearsal of procedures for solving particular classes of mathematics problems. In other words, fostering content orientation to mathematics rather than an expression orientation. Furthermore, the test, like the worksheet and tutorial, encourages inter-topic connectivity in that mathematics problems focus on more than one topic simultaneously and so require learners to select appropriate computational resources. The evaluative activity instantiated in the selection of mathematics problems for the test suggests an orientation to mathematics that is content oriented rather than expression oriented.

\section{Marking of Ivory College test (Sara and Jada)}

The memorandum of Common Test Functions 2012 provides solutions to the test problem as well as the allocation of marks. The memorandum appears very specific, as suggested by the comment 'must give co-ords' for Problem 1.5 and the details of how Problem 1.3 should be marked. It is interesting that in Problem 1.1, marks are allocated for factorising the quadratic equation and writing down the $x$-intercepts but not for setting up the equation, that is, for establishing $f(x)=0$ which is central to calculating the $x$-intercepts of the function. The mark allocation suggests evaluative criteria that prioritise obtaining the correct values of the $x$-intercepts without the notion of an equation serving as a computational resource. This hypothesis is borne out by the marking of the test scripts by Sara.

Sara mostly makes the evaluative criteria explicit to learners by correcting errors or providing evaluative commentary (see Figure 2), except for one learner's solution to Problem 1.6 (discussed later).

Sara awarded full marks for Problem 1.1 to two learners despite the fact that they generated the correct $x$-intercepts without setting up an equation (see Figure 2). Her marking of the learners' solutions as correct despite the explicit absence of an equation as a computational resource suggests an orientation to mathematics that is expression oriented rather than content oriented, that is, an orientation that focuses on producing the correct expressions irrespective of the underlying mathematical structures. However, the fact that she corrected the learner's solution indicates that she does make the evaluative criteria that the learners ought to display explicit.

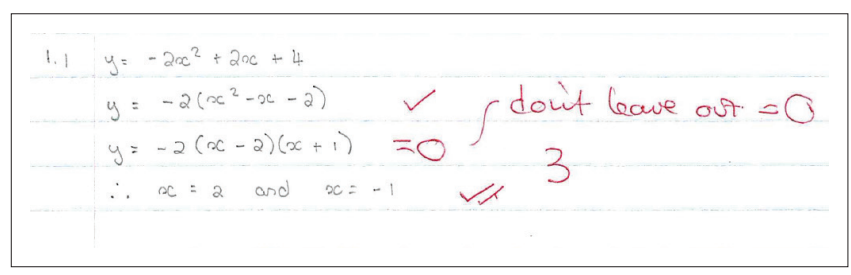

FIGURE 2: A learner's marked solution to Problem 1.1. 
Sara's marking of Problem 1.6 (see Figure 1) which focuses on a graphical solution of the inequality $f(x)<0$ (i.e. $-2 x^{2}+2 x+$ $4<0$ ) provides further evidence of a slippage towards expression orientation. Learner 1 produced ' $x<1$ and $x>2$ ' as the solution to Problem 1.6 which was marked as correct although the correct solution to Problem 1.6 is $x<-1$ OR $x>2$. Her failure to correct the learner's incorrect solution might be an oversight on her part given that her marking of learners' test scripts was generally consistent and she indicated that Learner 2's solution is incorrect by replacing 'AND' with 'OR'. As in the case of the marking of Problem 1.1, Sara awards full marks for Learner 2's incorrect solution to Problem 1.6. It thus appears that the correct usage of the logical connective 'OR' rather than 'AND' is not deemed important. It is curious though that Sara is not prepared to accept Learner 3's solution $(2<x<-1)$ as correct. Sara writes the following on the learner's script: 'you can't write this as one inequality. $2<-1$ is NOT true. $x>-2$ or $x<-1$ '. Note that Sara makes a mistake when she provides the corrected solution. She stated the solution is $x>-2$ or $x<-1$. The correct solution is $x>2$ or $x<-1$.

The statement $2<x<-1$ produced by Learner 3 is equivalent to the statement, $x<-1$ AND $x>2$, produced by Learner 2 and Learner 1 . Both statements imply that there is a number, $x$, which is simultaneously greater than 2 and less than -1 and so disrupt the order relation. However, the solution of Learner 1 is marked as correct with full marks without commentary and Learner 2's solution is awarded full marks but corrected.

Sara's marking of Learner 1's and Learner 2's solutions to Problem 1.6 suggests that the logical connectives and order relations are not explicitly required as computational resources and indicates that content that diverges both at the level of expression and at the level of content is accepted as correct. Furthermore, her marking indicates that the presence of the expressions $/ x<-1 /$ and $/ x>2 /$ are prioritised over the solution set that satisfies the condition that $f(x)<0$, suggesting an orientation to mathematics that is expression centred rather than content centred.

Jada's marking was consistent across learners' test scripts and she made the evaluative criteria explicit to learners by correcting errors or providing evaluative commentary (see Figure 3). In contrast to Sara, Jada deducted marks for errors committed by learners. For example, she deducted one mark for not equating $f(x)$ with 0 when solving Problem 1.1. She therefore prioritises the notion of an equation as a central computational resource in solving Problem 1.1. Her marking of learners' solutions stands in opposition to Sara's

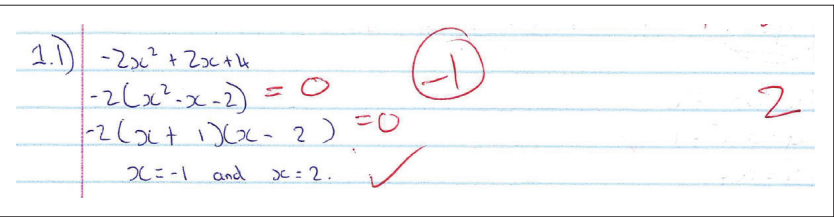

FIGURE 3: Noa's (P12) solution to Problem 1.1. marking and appears to be more content oriented than expression oriented.

\section{Arbor High test (Maya)}

At Arbor High, each Grade 10 teacher set their own mathematics test. The tests administered by the two Grade 10 teachers at Arbor High differed in terms of announced topics assessed. Maya's test covered the topics dealt with during the observed lessons and consisted of four test problems. Problems 1, 2 and 3 entailed finding the equation of a given function provided as a sketch (see Figure 4 for Problems 1 and 2) and Problem 4 focused on sketching the graph of the function $y=\frac{-3}{x+1}+2$.

Problems 1-3 are of the type 'calculate the equation of the function' and Problem 4 of the type 'sketch the graph of the function', which were the problem types covered in class during the observed lessons. All the test problems are classified as single-topic problems. The test problem types are the same as the problems used in the observed lessons, but the examples differed.

The test, like the problems used during the observed lessons, directly named the procedure that learners were expected to carry out. Maya's learners were not expected to analyse problems in order to select a particular procedure for solving a problem. Furthermore, learners in Maya's pedagogic context were not exposed to variations in the phrasing of problem types. For example, the 'calculate the equation of the linear function' mathematics problems provided by Maya all entailed a sketch with given intercepts. Variants of the same mathematics problem could, for example, be achieved through changing the nature of the given points: two intercepts or a $y$-intercept and any other point on the line or any two points on the line. Alternatively, the gradient of the line and another point could be provided.

The absence of problems that require analysis in order to select appropriate procedures for solving the problem and the lack of variation in problem statements are suggestive of an orientation to mathematics that attempts to elicit precise responses from learners through the rehearsal of particular procedures for solving particular problems. In other words,

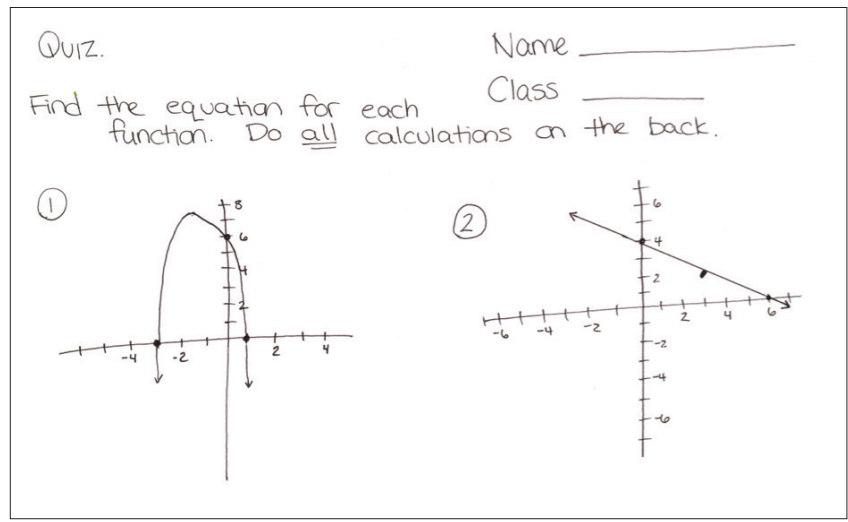

FIGURE 4: Extract of test on functions administered by Maya. 
learners are encouraged to recognise problem types and then select the correct procedure.

Furthermore, the test, like the problems used in the observed lessons, treated topics separately. Therefore, unlike the Ivory College test, the test set by Maya suggests a lack of inter-topic connectivity.

Only sketches of functions were provided in Problems 1, 2 and 3 of the test (see Figure 4), as was the case for the mathematics problems presented during the observed lessons. In other words, the mathematics problems did not state what type of function was represented nor was the general equation of the function provided. Learners were thus expected to determine the type of function from the sketch, suggesting that the expressive elements (the graphical expressions) serve as computational resources because it is the imagistic features of the text that learners are expected to draw on in order to determine the type of function represented. In other words, learners were expected to recognise the function type on the basis of the images (expressions) provided. The test problems and mathematics problems used in the observed lessons thus encourage an expression orientation to mathematics.

\section{Marking of Maya's test}

The total mark for the test and mark allocation per problem were not provided to learners and the teacher's memorandum did not show the mark allocation. From the marked scripts, it became apparent that the teacher allocated four marks per problem, bringing the total of the test to 16. In the marking of learners' test scripts, Maya at times indicated that an error was produced and deducted marks to penalise the learner. On a number of occasions though, errors produced by learners were not highlighted by the teacher and were marked as correct. An example is illustrated in Figure 5 which shows Learner 1's solution to Problem 1.

Learner 1 identifies the sketch as representing a parabola as indicated in her choice of general formula $y=a x^{2}+b x+q$. She produces the correct value for $a$ and although $b$ is correct, her solution indicates that the notion of an equation, central to the computation, is absent. She produces the expression $2-6=b$ from the expression $y=-2+b+6$, which is incorrect because $y$ simply 'disappears'. Despite the mathematical inconsistencies produced by Learner 1 , the teacher awards her full marks. So, Maya's evaluation of the learner's mathematical work reveals an orientation to

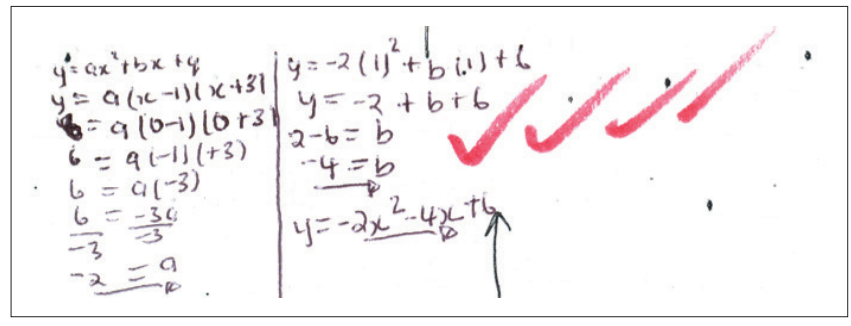

FIGURE 5: Learner 1's marked solution to Problem 1. mathematics that focuses on the correct expression in spite of mathematical violations.

Maya awards full marks to Learner 2 for her solution to Problem 1 despite a number of computational inconsistencies with respect to mathematics. The learner's final equation $y=-2 x^{2}-4 b+6$ is marked as correct even though it is incorrect presumably because she positioned the value of $b$ in the 'correct place' in the equation. After writing down the expected general formula $y=a x^{2}+b x+q$, the learner produced the expression $6=a(x+0)(x+6)$ which is incorrect but not pointed out by Maya. Later in the solution, the learner incorrectly computes the value of $a=\frac{6}{-3}=2$ but her value of $a$ is marked as correct by the teacher, presumably because the teacher assumes that the learner has merely made a computational error which is corrected later in her solution.

Maya's marking of the learner's solution suggests a strong expression orientation to mathematics since her assessment of learners' work validates the production of the expected expressions despite divergence from the mathematics content associated with the topic. Mathematics constituted in this pedagogic context is primarily a form of mathematical knowledge which diverges from mathematics at the level of expression and at the level of the content associated with the topic. The teacher's marking of learners' solutions suggests that her evaluation cultivates an orientation to mathematics that is expression oriented.

\section{Arbor High test (Jono)}

Jono's test (see Figure 6) comprises 10 problems requiring learners to 'find the domain and range' of functions, two of which are linear functions (Section A), three quadratic functions (Section B), three hyperbolic functions (Section C) and two exponential functions (Section D). The test is an extract of the worksheet used during the observed lessons and resembles the worksheet both in its structure and mathematics problems. In fact, the test constitutes a selection of items from the worksheet which is evident when we compare the test to the worksheet.

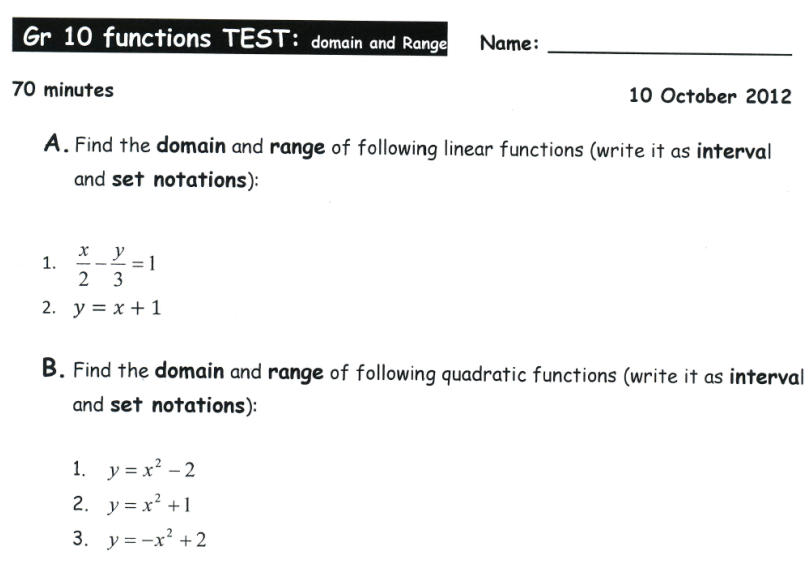

FIGURE 6: Extract of test on domain and range of functions administered by Jono. 
Test problem A1 corresponds with worksheet problem A5, test problem $\mathrm{A} 2$ with worksheet problem A1, test problems B1-B3 with worksheet problems B1-B3, test problems C1-C3 with worksheet problems $\mathrm{C} 1-\mathrm{C} 3$, test problem D1 with worksheet problem D1 and test problem D2 with worksheet problem D5. Thus, all the problems from the test were selected from the worksheet, sometimes in the same order.

All the test problems are classified as single-topic mathematics problems. Jono's learners, like Maya's learners, were not expected to analyse problems in order to select a particular procedure for solving a problem. The function type, for example linear functions or quadratic functions, was identified for the learner, thus generating a test of low complexity because learners mostly needed to recall the propositions with respect to each function type established during the observed lessons. For example, learners were expected to recall that the domain of a linear function is $\{x: x \in \mathbb{R} ;-\infty<x<+\infty\}$. Note that the inclusion of $-\infty<x<+\infty$ as part of the statement is really redundant but this was the legitimate response expected by Jono.

Jono's learners, like Maya's learners, were not exposed to variations in problem types. For example, mathematics problems related to domain and range of functions could be set in graphical form. In other words, learners have to deduce the domain and range from the graph of a function. The absence of problems that required analysis in order to select appropriate procedures for solving them and the lack of variation in problem statements suggest that learners were expected to rehearse and repeat particular procedures for solving particular problems, typical of an expression orientation to mathematics.

Furthermore, the learners had seen the test problems and worked through the problems in class. It seems that the test assesses whether learners are able to repeat the texts produced in class under test conditions. In other words, the evaluation encourages learners to reproduce texts that precisely conform with texts that are considered as legitimate in the pedagogic context, through repetition and rehearsal. The test is therefore strongly suggestive of an orientation to mathematics that is expression oriented.

\section{Marking of Jono's test}

The test memorandum provides solutions to the test problems but how marks ought to be awarded is not made explicit. The marked tests show that two marks were allocated per test problem, half a mark each for the domain and range expressed in set builder notation and in interval notation. The memorandum provided the domain for Problem A1 as $\{x: x \in \mathbb{R} ;-\infty<x<\infty\}$ in set builder notation and $(-\infty ; \infty)$ for interval notation. The range for Problem A1 was given as $\{y: y \in \mathbb{R} ;-\infty<y<\infty\}$ in set builder notation and for $(-\infty ; \infty)$ for interval notation. The interviewed learners' solutions to Problem A1 and Jono's marking of their solutions are shown in Table 1.

Table 1 shows (1) solutions to problem A1 (domain and range in set builder notation and interval notation) produced by learners who were selected to be interviewed, (2) whether the solution is correct or incorrect according to the teacher's memorandum and (3) the marks awarded by the teacher. We observe that incorrect components are treated as though they are correct. Ali produces incorrect statements for the domain and range in set builder notation but is awarded full marks by the teacher. Similarly, Ozi and Lea have three parts to the solution to problem A1 incorrect but Ozi is awarded 1.5 marks and Lea is awarded 1 mark. Similarly, Ory and Zoe both produce the correct domain and range in interval notation despite the fact that both learners obtained the incorrect set builder notation. Ory was awarded 1 mark and Zoe was awarded 1.5 marks.

Jono at times corrected errors and on other occasions he neglected to identify the errors. For example, inconsistencies are evident when we compare the marking of two learners' (Ozi and Ali) solutions to Problem A1. The set builder notation for the domain and range in both learners' solutions is incorrect as they violate order relations. Jono corrects Ozi's domain in set builder notation by putting rings around the inequality signs to indicate that they are incorrect and writing the correct expression $-\infty<x<\infty$ in place of the incorrect expression $-\infty>x>\infty$ but does not correct Ali's solution. Ali's statements for the domain as $\{x / x \in \mathbb{R} ;-\infty>x<\infty\}$ and range as $\{y / y \in \mathbb{R} ;-\infty>x<\infty\}$ for the function $\frac{x}{2}-\frac{y}{3}=1$ in set builder notation are incorrect but they are not corrected by the teacher. In fact, Ali makes the same mistake throughout

TABLE 1: Solutions to Problem A1 of Jono's learners selected to be interviewed.

\begin{tabular}{|c|c|c|c|c|c|c|}
\hline Name & Code & $\begin{array}{l}\text { Domain } \\
\text { (set builder) }\end{array}$ & $\begin{array}{l}\text { Domain } \\
\text { (interval) }\end{array}$ & $\begin{array}{l}\text { Range } \\
\text { (set builder) }\end{array}$ & $\begin{array}{l}\text { Range } \\
\text { (interval) }\end{array}$ & Mark awarded \\
\hline Tim & P01 & $\begin{array}{l}\{x: x \in \mathbb{R} ;-\infty<x<\infty\} \\
\text { correct }\end{array}$ & $\begin{array}{l}(-\infty ; \infty) \\
\text { correct }\end{array}$ & $\begin{array}{l}\{y: y \in \mathbb{R} ;-\infty<x<\infty\} \\
\text { correct }\end{array}$ & $\begin{array}{l}(-\infty ; \infty) \\
\text { correct }\end{array}$ & 2 \\
\hline Ali & P02 & $\begin{array}{l}\{x / x \in \mathbb{R} ;-\infty>x<\infty\} \\
\text { incorrect }\end{array}$ & $\begin{array}{l}(-\infty ; \infty) \\
\text { correct }\end{array}$ & $\begin{array}{l}\{y / y \in \mathbb{R} ;-\infty>x<\infty\} \\
\text { incorrect }\end{array}$ & $\begin{array}{l}(-\infty ; \infty) \\
\text { correct }\end{array}$ & 2 \\
\hline Ozi & P11 & $\begin{array}{l}\{x: x \in \mathbb{R} ;-\infty>x>\infty\} \\
\text { incorrect }\end{array}$ & $\begin{array}{l}(\infty ; \infty) \\
\text { incorrect }\end{array}$ & $\begin{array}{l}\{y: y \in \mathbb{R} ;-\infty>x>\infty\} \\
\text { incorrect }\end{array}$ & $\begin{array}{l}(-\infty ; \infty) \\
\text { correct }\end{array}$ & 1.5 \\
\hline Lea & P12 & $\begin{array}{l}\{x: x \in \mathbb{R} ; x \neq 0\} \\
\text { incorrect }\end{array}$ & $\begin{array}{l}(0 ; \infty) \\
\text { incorrect }\end{array}$ & $\begin{array}{l}\{y: y \in \mathbb{R} ;-\infty<x<\infty\} \\
\text { incorrect }\end{array}$ & $\begin{array}{l}(-\infty ; \infty) \\
\text { correct }\end{array}$ & 1 \\
\hline Ory & P17 & $\begin{array}{l}\{x: x \in z ; x<3\} \\
\text { incorrect }\end{array}$ & $\begin{array}{l}(-\infty ; \infty) \\
\text { correct }\end{array}$ & $\begin{array}{l}\{y: y \in \mathbb{R} ; y \geq 3\} \\
\text { incorrect }\end{array}$ & $\begin{array}{l}(-\infty ; \infty) \\
\text { correct }\end{array}$ & 1 \\
\hline Zoe & P18 & $\begin{array}{l}\{x: x \in \mathrm{R} ;-\infty ; \infty\} \\
\text { incorrect }\end{array}$ & $\begin{array}{l}(-\infty ; \infty) \\
\text { correct }\end{array}$ & $\begin{array}{l}\{y: y \in \mathbb{R} ; \neq 0\} \\
\text { incorrect }\end{array}$ & $\begin{array}{l}(-\infty ; \infty) \\
\text { correct }\end{array}$ & 1.5 \\
\hline
\end{tabular}


the test but Jono does not correct the error even once. The fact that Ali was awarded full marks for his solution to Problem A1 and the fact the Jono does not correct Ali's errors suggest that Jono does not require learners to use order relations as computational resources.

Jono's evaluative activity as instantiated in the marking of learners' test scripts validates content that differs from mathematics at the level of expression as well as the level of content. In addition, his evaluation of learners' test scripts represents an extreme version of expression orientation in that as long as the expressions produced by learners resemble the correct expression according to the memorandum they are accepted as correct.

\section{Concluding remarks}

Comparing the tests across the two schools reveals differences in the types of problems set in the two schools and differences in the preparation for tests and examinations. Ivory College learners' preparation involved classwork and independent work on worksheets and tutorials that pose mathematics problems in different ways. The types of problems encountered in class and independent work as well the test included both single-topic problems as well as multi-topic problems that required learners to draw on a number of different topics.

In contrast, Arbor High learners were only exposed to singletopic mathematics problems in their tests which were restricted to mathematics problems encountered during the observed lessons with no variation in the way problems are phrased. The Arbor High tests appear to encourage an expression orientation given the similarity of the mathematics problems used in the tests to those used during the observed lessons, with Jono's test representing an extreme case of expression orientation because the test problems were extracted from the worksheet used during the observed lessons. The tests suggest that rehearsal of model texts in the form of set solution procedures for set problem types is the primary mode of mathematics reproduction in the Arbor High pedagogic contexts.

The absence of multi-topic mathematics problems in the Arbor High tests corresponds with the absence of multi-topic mathematics problems in the observed lessons, which indicates that topics are treated in isolation by Arbor High teachers thus resulting in a lack of inter-topic connectivity. As such, Arbor High learners are left to make connections between topics independently of the teacher. It could be argued that synthesis of school mathematics topics into a coherent whole is made much harder for the learners from working-class backgrounds than the learners from upper-middle-class or elite backgrounds.

In addition, Ivory College learners' exposure in class and in tests to multi-topic problems which resemble examination type problems means that they appear to be better prepared with the support of their teachers for more mathematically demanding problems than their counterparts at Arbor High.
It appears that in the working-class contexts, learners are left to make connections to topics and tackle more complex problems on their own, that is, without instruction and support from teachers, which perhaps provides insight into why mathematics performance for the majority of secondary learners in South Africa is so poor.

Comparing the marking of the test also reveals differences across the four pedagogic contexts. Maya and Jono's marking included instances where mathematical violations were not corrected by the teacher and were accepted as correct. Furthermore, their marking is inconsistent and at times learners' solutions marked as correct did not match their memoranda. Sara and Jada corrected learner errors, thus making learners' errors explicit to them. Sara, however, at times made marking errors and did not deduct marks even though the solutions contained errors and on one occasion marked an incorrect solution as correct. Thus, Jono's and Maya's marking encourages an expression orientation to mathematics. Jono's and Maya's marking confirms the expression orientation observed in the observed lessons (see Jaffer, 2018). Sara's marking reveals aspects of expression orientation that is reminiscent of the marking in the working-class contexts although to a lesser degree. The types of problems learners are exposed to suggest an orientation that is more content centred. Although not discussed here, the orientation to mathematics evident in the observed lessons taught by Sara was more content oriented (see Jaffer, 2018). Thus, Sara presents a case of a hybrid of expression orientation and content orientation.

We, therefore, see differences as well as similarities in the pedagogic practices across social class contexts, disrupting entrenched narratives in the literature about stark differences between schools populated by learners from working-class backgrounds and schools populated by learners from upper-middle-class or elite backgrounds. The crucial difference, however, does appear to hinge on the absence of content orientation in the school populated by learners from working-class backgrounds and the extensive preparation and type of problems that learners in the upper-middle-class or elite context are exposed to, which perhaps provides a possible explanation for the persistent differential performance in mathematics along the lines of social class.

\section{Acknowledgements}

This article derives from my doctoral research project. I hereby thank my supervisors, Associate professor Zain Davis and Professor Emeritus Paula Ensor, for their guidance and support during the supervision of my PhD study. I further wish to thank both supervisors for their valuable comments on a draft version of this article.

\section{Competing interests}

I declare that I have no financial or personal relationships that may have inappropriately influenced me in writing this article. 


\section{Authors' contributions}

I declare that I am the sole author of this article.

\section{Ethical consideration}

Ethical clearance for this research was obtained from the Research Ethics Committee of the University of Cape Town in the Western Cape (ethical clearance number EDNREC202000909). Ethical issues including anonymity, confidentiality and voluntary participation were discussed with participants in the study and written consent for participation in the research was obtained from participants

\section{Funding information}

This work is based on research supported in part by the National Research Foundation of South Africa under grant number 92639. The research also benefited from funding from the university's research capacity initiative. Any opinion, findings, conclusions and recommendations expressed here are those of the author and are not necessarily attributable to either of these organisations.

\section{Data availability statement}

Data sharing is not applicable to this article.

\section{Disclaimer}

The views and opinions expressed in this article are those of the author and do not necessarily reflect the official policy or position of any affiliate agency of the author.

\section{References}

Anyon, J. (1980). Social class and the hidden curriculum of work. Journal of Education, 162, 67-92. https://doi.org/10.1177/002205748016200106

Anyon, J. (1981). Social class and school knowledge. Curriculum Inquiry, 11(1), 3-42. https://doi.org/10.1080/03626784.1981.11075236

Atweh, B., Bleicher, R., \& Cooper, T. (1998). The construction of the social contex of mathematics classrooms: A sociolinguistic analysis. Journal for Research in Mathematics Education, 29(1), 63-82. https://doi.org/10.5951/ jresematheduc.29.1.0063

Atweh, B., \& Cooper, T. (1995). The construction of gender, social class and mathematics in the classroom. Educational Studies in Mathematics, 28(3), 293-310. https:// doi.org/10.1007/BF01274178

Bernstein, B. (1990). Class codes and control. Volume 4: The structuring of pedagogic discourse. London: Routledge.

Bernstein, B. (1996). Pedagogy, symbolic control, and identity: Theory, research, critique. London: Taylor \& Francis.

Bernstein, B. (2000). Pedagogy, symbolic control, and identity: Theory, research, critique. Revised edn. Oxford: Rowman \& Littlefield.

Boaler, J. (1998). Open and closed mathematics: Learner experiences and understandings. Journal for Research in Mathematics Education, 29(1), 41-62. https://doi.org/10.5951/jresematheduc.29.1.0041

Brown, A., \& Dowling, P. (1998). Doing research/reading research: A mode of interrogation for education. London: Falmer Press.

Carnoy, M., Chisholm, L., Chilisa, B., Addy, N., Arends, F., Baloy, H., ... Tsheko, N. (2011) The quality of mathematics teaching in North-West Province. Technical Report Stanford, CA: Stanford University Human Sciences Research Council.

Chevallard, Y. (1989). On didactic transposition theory: Some introductory notes. Proceedings of the international symposium on selected domains of research and development in mathematics education (pp. 51-62). Bratislava, 3-7 August 1988.

Chisholm, L., \& Sujee, M. (2006). Tracking racial desegregation in South African schools. Journal of Education, 40(1), 141-159.

Chitsike, M. (2011). An investigation of the co-constitution of mathematics and learne identification in the pedagogic situations of schooling, with special reference to the teaching and learning of mathematics in a selection of grade 10 mathematics lessons at five schools in the Western Cape Province of South Africa. Unpublished M.Ed. Dissertation. University of Cape Town.
Chomsky, N. (2009). Cartesian linguistics: A chapter in the history of rationalist thought (3rd edn.). Cambridge: Cambridge University Press. https://doi. org/10.1017/CBO9780511803116

Cooper, B., \& Dunne, M. (2000). Assessing children's mathematical knowledge: Social class, sex, and problem-solving. Oxford: Open University Press.

Davis, Z. (2005). Pleasure and pedagogic discourse in school mathematics: A case study of a problem-centred pedagogic modality. Unpublished PhD thesis, University of Cape Town.

Davis, Z. (2011). Orientations to text and the ground of mathematical activity in schooling. In H. Venkat \& A. Essien (Eds.), Proceedings of the 17th Nationa Congress of the Association for Mathematics Education of South Africa (pp. 310-322). University of the Witwatersrand, Johannesburg, 11 July.

Davis, Z. (2013). The use of the idea of coherence in descriptions and analyses of school mathematics curricula, textbooks and pedagogy. (Plenary address to the 19th AMESA Congress.) In Z. Davis \& S. Jaffer (Eds.), Proceedings of the 19th Annual Congress of the Association for Mathematics Education of South Africa (Volume 1, pp. 35-46). University of the Western Cape, Cape Town, 24-28 June.

Davis, Z., \& Ensor, P. (2018). Pedagogic modalities and the ritualising of pedagogy. In B. Barret, U. Hoadley, \& J. Morgan (Eds.), Knowledge, curriculum, equity: Socia realist perspectives (pp. 200-218). London: Routledge. https://doi.org/ 10.4324/9781315111360-13

Department of Basic Education. (2011). Curriculum and assessment policy statement grades 10-12 Mathematics. Pretoria: Department of Basic Education (DBE).

Dowling, P. (1998). The sociology of mathematics education: Mathematical myths/ pedagogic texts. London: Falmer Press. Francis.

Eco, U. (1976). A theory of semiotics. Bloomington, IN: Indiana University Press.

Feza, N. (2012). Language: A cultural capital for conceptualizing mathematics knowledge. International Electronic Journal of Mathematics Education, 7(2), 62-79.

Franklin, S. (2017). Education rights in independent schools. In F. Veriava, A. Thom, \& T. Hodgson (Eds.), Basic education rights handbook: Education rights in South Africa (pp. 353-372). Johannesburg: Section 27.

Graven, M. (2014). Poverty, inequality and mathematics performance: The case of South Africa's post-apartheid context. ZDM, 46, 1039-1049. https://doi. org/10.1007/s11858-013-0566-7

Graven, M., \& Venkat, H. (Eds.) (2017). Improving primary mathematics education teaching and learning: Research for development in resource-constrained contexts. London: Springer Nature. https://doi.org/10.1057/978-1-137-52980-0

Hoadley, U. (2005). Social class, pedagogy and the specialisation of voice in four South African Primary Schools. Unpublished PhD thesis, University of Cape Town.

Hoadley, U. (2007). The reproduction of social class inequalities through mathematics pedagogies in South African primary schools. Journal of Curriculum Studies, 39(6) 679-706. https://doi.org/10.1080/00220270701261169

Jaffer, S. (2010). An investigation into orientations towards privileged texts in Grade 8 mathematics classrooms. In V. Mudaly (Ed.), Proceedings of the 18th Annual Meeting of the Southern African Association for Research in Mathematics, Science and of the Southern African Association for Research in Mathematics, Science
Technology Education - Crossing the Boundaries. UKZN, Durban, 18-21 January.

Jaffer, S. (2012). The notion of 'topic' in school mathematics and the problem of isotopic coherence. In S. Nieuwoudt, D. Laubscher, \& H.E. Dreyer (Eds.) Proceedings of the 18th National Congress of the Association for Mathematical Education of South Africa (AMESA) (pp. 178-185). University of the North-West, Potchefstroom, 24-28 June.

Jaffer, S. (2018). Pedagogic evaluation, computational performance and orientation to mathematics: A study of the constitution of Grade 10 mathematics in two secondary schools. Unpublished PhD thesis, University of Cape Town.

Jaffer, S., \& Davis, Z. (2012). Investigating the relation between top-performing Grade 10 learners' elaborations of school mathematics and their social class membership. In D. Nampota \& M. Kazima (Eds.), Proceedings of the 20th annual meeting of the Southern African Association for Research in Mathematics, Science and Technology Education, Volume 1: Long Papers (pp. 105-118). University of Malawi, Cross Roads Hotel, Lilongwe, Malawi, 16-19 January.

Kaiser, G., \& Schwarz, B. (2010). Authentic modelling problems in mathematics education - Examples and experiences. Journal für Mathematik-Didaktik, 31, 51-76. https://doi.org/10.1007/s13138-010-0001-3

Kaur, B. (2010). A study of mathematical tasks from three classrooms in Singapore. In Y. Shimizu, B. Kaur, R. Huang, \& D. Clarke (Eds.), Mathematical tasks in classrooms around the world (pp. 15-34). Rotterdam: Sense Publishers. https://doi org/10.1163/9789460911507_003

Lotman, Y.M. (1990). Universe of the mind: A semiotic theory of culture. Bloomington, IN: Indiana University Press.

O'Halloran, K. (2004). Discourses in secondary school classrooms according to socia class and gender. In J.A. Foley (Ed.), Language, education and discourse. Functiona approaches (pp. 191-225). London: Continuum.

Reddy, V., Van der Berg, S., Lebani, L., \& Berkowitz, R. (2006). A trend analysis of Matric maths. In V. Reddy (Ed.), Marking matric colloquium proceedings. Cape Town: HSRC Press.

Schollar, E. (2008). The primary mathematics research project (2004-2007). Towards evidence-based educational development in South Africa. A final project report. Johannesburg: Eric Schollar and Associates Cc.

Shimizu, Y., Kaur, B., Huang, R., \& Clarke, D. (Eds). (2010). Mathematical tasks in classrooms around the world. Rotterdam: Sense Publishers. https://doi org/10.1163/9789460911507

Spaull, N. (2019). Equity: A price too high to pay? In N. Spaull \& J. Jansen (Eds.), South African schooling: The enigma of inequality. Policy implications of research in African schooling: The enigma of inequality. Policy implications of research in
education (volume 10). Cham: Springer. https://doi.org/10.1007/978-3-030education $18811-5$ 
Spaull, N., \& Kotze, J. (2015). Starting behind and staying behind in South Africa. The case of insurmountable learning deficits in mathematics. International Journal of Educational Development, 41, 13-24. https://doi.org/10.1016/j. ijedudev.2015.01.002

Swanson, D. (2002). 'Disadvantage' and school mathematics: The Politics of context International Journal of Learning, 9, 1471-1480.

Swanson, D. (2006). Power and poverty - Whose, where, and why?: Schoo mathematics, context and the social construction of 'disadvantage'. In J. Novotná, H. Moraová, M. Krátká, \& N. Stehlíková (Eds.), Proceedings 30th Conference of the International Group for the Psychology of Mathematics Education (Vol. 5, pp. 217-224). Prague: PME.
U.S. Department of Education. (2003). Teaching mathematics in seven countries: Results from the TIMSS 1999 video study. NCES 2003-013 Revised, By James Hiebert, Ronald Gallimore, Helen Garnier, Karen Bogard Givvin, Hilary Hollingsworth, Jennifer Jacobs, Angel Miu-Ying Chui, Diana Wearne, Margaret Smith, Nicole Kersting, Alfred Manaster, Ellen Tseng, Wallace Etterbeek, Carl Manaster, Patrick Gonzales, James Stigler. Washington, DC: National Center for Education Statistics.

Yin, R.K. (2009). Case study research: Design and methods (4th edn.). Thousand Oaks, CA: Sage.

Yudell, M., Roberts, D., DeSalle, R., \& Tishkoff, S. (2016). Taking race out of human genetics. Science, 351(6273), 564-565. https://doi.org/10.1126/science.aac4951 